\title{
Lung ultrasound: a narrative review and proposed protocol for patients admitted to Cardiac Rehabilitation Unit
}

\author{
Dario Tino Bertolone, Cristina De Colle, Francesco Rozza, Ilaria Fucile, Ciro Santoro, Maurizio Conte, \\ Nicola De Luca, Costantino Mancusi \\ Hypertension Research Center, Department of Advanced Biomedical Science, Federico II University Hospital, Naples, Italy
}

\begin{abstract}
Lung ultrasonography (LUS) has become in the last 10 years a technique that has reduced the need of second level diagnostic methods such as chest X-ray (CXR) and computerize tomography (CT) for the diagnostic imaging of lung and pleural space, throughout its diagnostic accuracy, radiation free, low cost, real time and bedside approach. The common use of LUS has been recently extend to cardiac and pulmonary disease even in context of Cardiac Rehabilitation Unit and it could be an additional tool for physiotherapist for the management of patients during Rehabilitation course. The authors performed a literature review in PubMed and suggested a new standardize protocol for LUS, based on guidelines and expert consensus document, for patients admitted to Cardiac Rehabilitation Unit. In this protocol, LUS should be performed in six scan each hemithorax, covering twelve imagine regions. For each scan will be noted a specific physiologic or pathological patterns. Furthermore, we suggest for each patient, the use of the Lung Ultrasound Score (LUS score) to obtain a global view of lung aeration and to monitor any changes during the hospitalization. An increase in score range indicates a more severe condition. This Lung
\end{abstract}

Correspondence: Costantino Mancusi, MD, Department of Advanced Biomedical Science, Federico II University Hospital, Via Pansini 5 80131, Naples, Italy.

Tel. +39.08.17462013.

E-mail: costantino.mancusi@unina.it

Key words: Dyspnea; heart failure; inferior vena cava; pleural effusion.

Conflict of interest: The authors declare that they have no competing interests, and all authors confirm accuracy.

Ethics approval and consent to participate: Not applicable.

Received for publication: 28 December 2020.

Accepted for publication: 26 June 2021.

${ }^{\circ}$ Copyright: the Author(s), 2021

Licensee PAGEPress, Italy

Monaldi Archives for Chest Disease 2022; 92:1753

doi: 10.4081/monaldi.2021.1753

This article is distributed under the terms of the Creative Commons Attribution Noncommercial License (by-nc 4.0) which permits any noncommercial use, distribution, and reproduction in any medium, provided the original author(s) and source are credited.
Ultrasonography Protocol should be performed in all patients at the time of admission to Cardiac Rehabilitation Unit to monitoring the aeration of the lungs and the possible lung and/or pleura complications after a cardiac disease avoiding the use of second level surveys.

\section{Introduction}

Lung ultrasonography (LUS) has become a widely accepted diagnostic tool during the last 10 years, due to its increasing availability, accuracy and awareness among different specialists. Lung ultrasound allows physicians to easily examine lungs and pleural space in the context of critically ill patients. Its application reduces the need of standard chest X-ray (CXR) and computerized tomography (CT). Its repeatability, reliability, make LUS an efficient modality to rapidly scan the lung and pleura at the bedside in a confident and cost benefits approach without radiation exposure risk [1]. Modern lung ultrasound is applied not only in emergency and critical care subset but also in pulmonary and cardiovascular diseases [2].

This review focuses on LUS, summarizing its applications for the evaluation and management of patients admitted to Cardiac Rehabilitation Unit.

\section{Methods}

Literature review was performed in PubMed. The search terms included, "lung ultrasound", "lung ultrasound in cardiac surgery", "lung ultrasound in heart failure", "lung ultrasound and cardiology", "lung ultrasound and cardiac rehabilitation unit". Levels of evidence as far as mentioned in this article refer to the international evidence-based recommendations for pointof-care ultrasound published in 2012 and to the Expert consensus document "Reporting checklist for quantification of pulmonary congestion by lung ultrasound in heart failure" published in 2019 .

\section{LUS: technical and terminology}

According to the "International evidence-based recommendations for point-of-care lung ultrasound", different transducers can be used for thoracic ultrasonography depending on the structures to be displayed: for visualizing deep structures is 
referred a probe designed for abdominal ultrasonography like convex or micro-convex probe, instead for superficial structures and for a more detailed visualization of the pleural line is referred a linear probe. However, a phased array probe aimed for cardiac imaging, can also be used for thoracic ultrasonography, thus leading to a reduction in costs, being able to achieve two purposes with a single probe [3]. For a prompt and straightforward evaluation, a compact portable [4] or handheld unit with a micro-convex probe may be used (evidence level B) [5]. There is no mandatory technique to perform lung ultrasound, however the examination should be conducted in a sitting patient or placed at 45 degrees, thus allowing the evaluation of posterior recesses. In all those conditions where this is not possible, the scan can be performed in a supine position, at rest, tilting the patient from the opposite side to the one of interest, in order to get a better view of lateral and posterior recesses. Scans are captured by placing the probe through the intercostal spaces. The main limitations are represented by the ribs and the heart.

In order to perform a complete and detailed examination 12 images should be acquired, 6 for each side. For this reason each hemithorax is divided into anterior, lateral, and posterior areas by the anterior and posterior axillary lines. Imaging is performed in the upper and lower part of each of these regions [6]. The use of a shorter protocol is recommended for specific clinical indications and emergency situations [7-8]. The most common approach on international consensus omits the posterior scan regions and uses only the anterior and posterolateral chest wall approaches in supine position. The approach suggested by Lichtenstein in his BLUE protocol is to perform two anterior scans called BLUE points [9] (BLUE = bedside lung ultrasound in emergency) and one posterolateral scan called PLAPS point [10-11] (PLAPS = posterolateral alveolar and/or pleural syndrome) (Figure1).

The first sign needs to be detected is the pleural line. Owing to a reflection phenomenon, the ultrasound wave is mirrored at the pleural line due to the differences in impedance between alveolar air and soft tissues of the thoracic wall. Hence, the pleural line is visualized as a hyperechoic horizontal line. Physiologically, inspiration and expiration lead to a lung volume change and consequently a dynamic horizontal movement of the visceral pleural surface on the parietal pleura. This movement, synchronous with the respiration, is called "lung sliding". The evidence_of "lung sliding" indicates preserved lung ventilation and direct contact of the visceral pleura to the parietal pleura, for the exclusion of air inside the pleural layer. The space delimited by the pleural line, silhouette of the ribs and the bottom of the image is called Merlin's space: it indicates a space where the back-scattering of ultrasound waves shows a healthy aerated lung tissue [12]. The use of M-mode to record lung sliding in healthy subject shows that the tissues above the pleural line remain static during respiration producing parallel lines described as "waves", whereas artifacts below the pleural line appears speckled, like sand. Since this image remember calm waters (structures above the pleural line) moving on the sandy beach (structures below pleural line), it was called "seashore sign".

Lung ultrasound is an artefacts study, whose originate from the interaction between ultrasound waves with the air contained within the pulmonary parenchyma [13]. An example of these artifacts resulting from several reflections of ultrasound waves on the pleural line, are called "A lines": single or multiple horizontal lines, parallel to the pleural line, visible within Merlin's space [14].

Another type of artifacts, called "B-lines" or "B-pattern", arise from the pleural line as long hyperechoic, well-defined and obliterate vertical reverberations that follow lung sliding during respiration. These findings may indicate congestive or fibrotic changes in the interstitial space, being pathognomonic of "interstitial syndrome", as described later in the text. These artifacts are also called "comet tail" for their feature [15].

Another kind of comet-tail artifacts are the Z-lines: vertical like the B lines they arise from the pleural line but are ill-defined, vanishing after a few centimeters $(2-5 \mathrm{~cm})$, not erasing the A lines, and independent from lung sliding. It could be observed in normal subjects as well as in cases of pneumothorax [16].

Another important sign found in lung ultrasound studies is the "curtain sign". In normal LUS the "curtain sign" is seen at the posterior costophrenic recess of the thorax and is a dynamic sign in concert with the respiratory movement. In fact, during inspiration the lung expands into the costophrenic recess and the inferior edge of the lung appears to move downwards, covering a portion of the intraabdominal structure, such as liver and spleen. The lung overlap into the abdomen creates a bounded leading edge of the air artifact, giving the impression of a lung curtain [17].

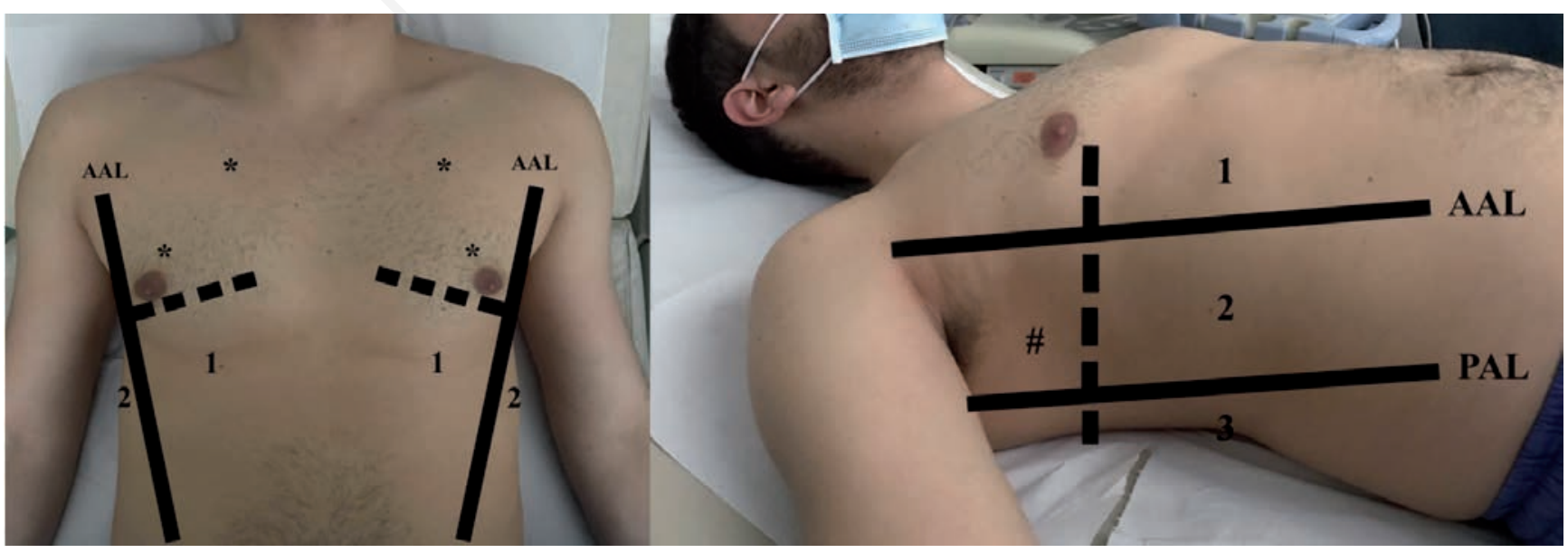

Figure 1. Lung ultrasound examination. AAL, anterior axillary line; PAL, posterior axillary line; 1 - 3, anterior, lateral and posterior scanning zones (each divided into upper and lower part by the dotted line). Asterisks: upper and lower BLUE points; hashtag: PLAPS point. 


\section{LUS: main application in cardiology}

Admission to Cardiac Rehabilitation Unit is indicated following acute coronary syndrome, coronary and valvular heart surgery, in chronic ischemic heart disease, in post-acute and chronic decompensated heart failure (HF) [18]. In these clinical setting different complications may occur affecting lung dynamics, such as Community Acquired Pneumonia (CAP), lung interstitial syndrome due to heart failure, pneumothorax due to Pace Maker or ICD implantation [19].

Lung ultrasound is a useful tool to detect these complications with a sensitivity and specificity comparable to CXR and CT, representing a pivotal technique for the diagnostic and therapeutic management of these patients [20]. The following sections analyze the main possible application of LUS in Cardiac Rehabilitation Unit.

\section{LUS: interstitial syndrome}

According to the 2019 European Society of Cardiology (ESC) Expert Consensus Document [21] LUS has been proposed as a suitable method for the evaluation of patients with acute and chronic HF. There has been an exponential increase in clinical application of lung ultrasound for the detection of pulmonary congestion in HF. This technique allows the assessment of pulmonary congestion in patients presenting with acute dyspnoea with higher accuracy and sensibility compared to chest semeiotics or CRX. The prompt findings of pulmonary congestion as "B-lines", permit to start promptly the treatment of acute HF and is helpful to lend prognostic information in both acute and chronic HF [22].

By definition, interstitial syndrome is characterized by the presence of bilateral and symmetrical B lines on LUS examination. Several clinical conditions such as acute cardiogenic pulmonary edema, interstitial pneumonia, or acute respiratory distress syndrome may present with this sonographic pattern [23].

The most specific sonographic signs for the evaluation of an interstitial syndrome in patients with acute pulmonary edema are:

- Assessment of the affected lung fields: in patients with acute pulmonary edema, interstitial syndrome is arranged in a homogeneous way, affecting both anterior and posterior lung fields.

- B-lines can be bilateral and symmetrical, and in more severe conditions merged, achieving the so-called "white lung" pattern, that indicates "wet lung", in which the increase of extravascular lung water exceeds the amount of aerated tissue. In this case, the physiological A-lines, expression of normal aerated lung, will not be present. A scoring system has been edited to define a zone as 'positive' for pulmonary congestion, in which a minimum number of B-lines in each lung region is used. Positive zones are then summed to define a cut-off value. For example, $\geq 3$ B-lines in two zones or more on each hemithorax delineate the diagnosis of pulmonary oedema in dyspnoeic patients [24,25].

Lung sliding is preserved, with thin and regular pleural line.

- In general, the anterior lung fields may be less affected, due to the gravitational way by which the extravascular water is distributed, but no "spared areas" are found.

Small consolidations can be observed, especially in posterior basal lung fields.

Pleural effusions are common. According to ESC guidelines for the diagnostic and treatment of acute and chronic heart failure, in up to $20 \%$ of patients with acute HF, CXR could appear normal due to the less sensitivity of this method compared to LUS, particularly for mild to moderate pleural effusions [26].

\section{LUS: Pleural effusion after cardiac surgery}

Patients undergoing cardiac surgery may run into several pulmonary complications. The causes of these complications include the side effects of general anesthesia, median sternotomy, cardiopulmonary bypass, internal mammary artery harvesting, heart failure, infection, and mechanical ventilation and have a strong impact on the mortality and morbidity of these patients. After coronary artery bypass graft operation up to $95 \%$ of patients may present pleural effusion [27].

Indeed, pleural effusion is the most widespread pulmonary complication, and on LUS examination it can be identified as an anechoic area in the pleural space. The "quad sign" represents the quadrangular space in which the pleural effusion appears bounded by the parietal pleura, the ribs and the visceral pleura (identified by the pulmonary line).

With the use of M-mode is detect a characteristic dynamic sign, called "sinusoid sign". This finding is typical of free and non-organized fluids and demonstrates the physiological movement of the respiratory cycle between the parietal pleura (pleural line) and visceral pleura (lung line). The distance between the two pleural layers decreases in inspiration due to the increase in chest volume and increases in expiration. LUS can detect even small amounts of pleural effusion (up to a minimum of $5 \mathrm{~mL}$ ), which cannot be identified using chest radiography. Actually, lung ultrasound is more accurate in detecting pleural effusion than supine radiography and is as accurate as CT [28]. The amount of pleural effusion is quantifiable measuring the maximal expiratory interpleural distance from the pleural line to the lung line on the posterior axillary line with the patient in the supine position or the paravertebral, scapular, posterior axillary, or medial axillary lines when the patient is in the sitting position. Balik et al. proposed a formula to determine the volume of free fluid in the pleural cavity: the distance $(\mathrm{mm})$ of the pleural layers from the posterior axillary line at the end of expiration multiplied by 20 is used to calculate the pleura effusion volume $(\mathrm{mL})$ [29]. The amount of pleural effusion can be scored as small ( 2 to $15 \mathrm{~mm}$ ), moderate ( 15 to 25 $\mathrm{mm}$ ), or large $(>25 \mathrm{~mm})$. Effusions $<2 \mathrm{~mm}$ are meaningless and are not considered. This formula shall not be used for contained and organized effusions. LUS may also determine the nature of the effusion. Transudates appear as anechoic fluids, while exudates or hemothorax appear as fluids with mobile echoes inside the effusion or hyperechoic structures (fibrinous septa) [30] (Figure 2). However, when faced with an anechoic effusion, the only way to differentiate between transudate and exudate is to use thoracentesis [31]

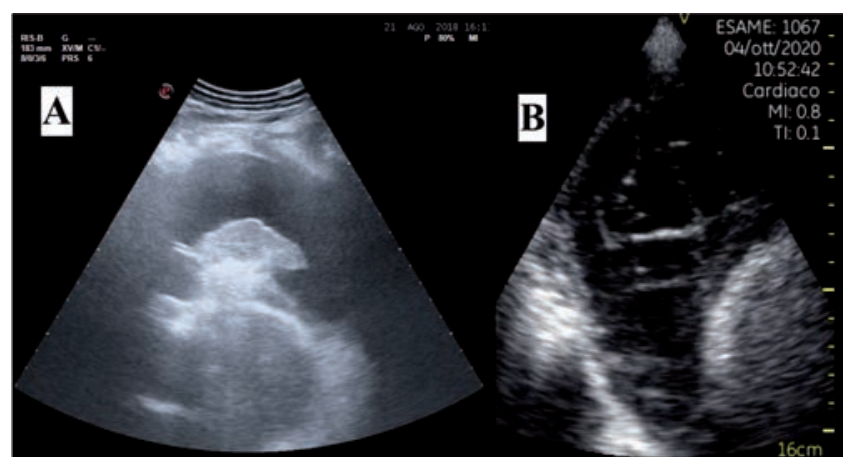

Figure 2. A) Massive pleural effusion (trasudate) with lung collapsed. B) Fibrinous septa in the form of hyperechoic structures within pleural effusion. 
In case of massive pleural effusion and related totally lung collapsed, it's likely to detect, when examining the left hemithorax, the cardiac chambers, pericardium and the cross-section aorta, normally covered by the lung. This scan is called "Heart-Lung Window" (Figure 3).

\section{LUS: pneumothorax as complication of PMK or ICD/CRT implantation}

Pneumothorax as a result of PMK or ICD/CRT implant is usually an early complication (up to $0.7-1.7 \%$ ) during cannulation of the subclavian or axillary vein and it mostly happen on the same side of venous access [32]. LUS is superior for detection of pneumothorax when compared to antero-posterior CXR, having a sensitivity of $78.6 \%$ and a specificity of $98.4 \%$ [33], both higher than X-ray.

Evaluation of patients with pneumothorax requires the assessment of the most superficial lung fields, as the anterior and lateral regions, because due to gravity the air is arranged in the upper fields in patient in supine position. It is also important to research several ultrasonographic signs including lung sliding, lung pulse, lung point, and B-lines also with the support of M-mode [34].

The sonographic signs of pneumothorax include:

- Presence of lung points: a lung point is found at the crossing point between the partially collapsed lung and the air-filled pneumothorax space, and is a dynamic finding, since the lung, although partially collapsed, continues to expand with the respiratory movements. The lung point is $100 \%$ specific but only $60 \%$ sensitive for pneumothorax. In supine position, the location of the lung point gives a semi-quantitative estimation of the extension of the pneumothorax. In a spread pneumothorax, the lung point is found in the lateral and posterior lung fields. If the pneumothorax is small, the lung point will be found in the anterior pulmonary regions. The lung point will be absent in case of massive pneumothorax as the lung will be completely retracted to the hilum [35].

- Absence of lung sliding: consisting in no sliding of pleural leaflets. The presence of lung sliding indicates that the visceral pleura flows over the parietal pleura and therefore the lung is on the chest wall. In pneumothorax, air in the pleural space prevents the two leaflets from flowing. Its absence has a sensitivity of $95.3 \%$, specificity of $91.1 \%$, and negative predictive value of $100 \%(p<0.001)$ for pneumothorax with a low falsepositive rate [36]. With the aid of $M$-Mode, an additional finding may be the so-called "stratosphere sign" or "barcode sign", which indicates the absence of pleural movement resulting in a static pattern and that replaces the physiological "seashore sign".

- Absence of B-lines

- Absence of lung pulse

In 1995, Lichtenstein et al. proposed a flow chart to rule in or rule out pneumothorax evaluating specific ultrasonographic findings reviewed in 2019 by Copetti et al. (Figure 4).

\section{LUS: Pneumonia}

The diagnosis of pneumonia can never be obtained by thoracic imaging alone (LUS, X-Ray or CT) but requires the integration of other essential parameters such as clinical history of the patient, physical examination and laboratory results. LUS is considered a useful technique in the diagnostic work up of pneumonia, even more accurate than CXR in expert hands even though chest CT remains the "gold standard" [37].

The ultrasound sign that best characterizes pneumonia is the presence of lung consolidations

Lung consolidation is characterized as an echo-poor region or a tissue-like structure located in the subpleural space. The lung with a spread consolidations appears with a tissue-like pattern reminiscent of the liver, so called "lung hepatization". The consolidations can occupy an entire lobe or extend into focal or multifocal areas (translobar or non-translobar), or just limited to an area below the pleural line [38].

- On colorDoppler ultrasonography, the vessels surrounding the consolidations have regular course and the circulation is equally ramified.

- Presence of air broncograms: these are point-like or linear, hyperechoic structures within the consolidation area, static or dynamic as a result of respiratory acts. A dynamic air bronchogram is characteristic of pneumonia [39].

- Presence of fluid broncograms: branched or tubular, hypoechoic/anechoic structures. They could be visible in early stages of pneumonia and are characteristic of post obstructive pneumonia [40].

- B-lines may be associated with pneumonia and can be, depending on the severity of the disease, sub-segmental, segmental, lobar, multifocal, or generalized distribution. They are mainly characteristic of interstitial pneumonia [41].

Bacterial pneumonias may evolve in necrosis and abscesses that appear as round or oval and largely anechoic lesions within the consolidated lung. The lung abscess may have an echogenic margin and contain an air artefact within.

Moreover, LUS ensures the monitoring of aeration changes in patients with Community-Acquired Pneumonia and the follow-up

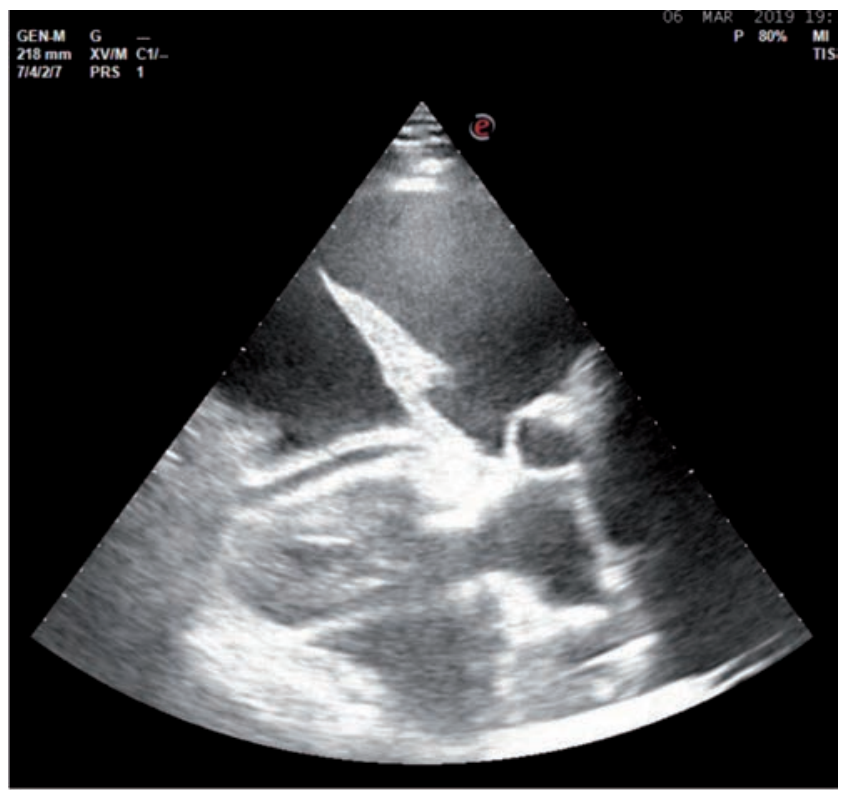

Figure 3. Heart lung window. From the top to the bottom: massive pleural effusion; lung totally collapsed; pericardial effusion; cross-section aorta and cardiac chambers. 
of the effects of therapy, better than CXR and CT particularly in terms of saving the patient from radiation exposure, delaying or avoiding transportation to the radiology unit, and guiding lifesaving therapies [42].

\section{Lung ultrasound during physic rehabilitation}

In the last decade the use of LUS in the management of critical care patients by physiotherapists has gradually gained great importance [43], not only in the setting of musculoskeletal rehabilitation, but especially in terms of respiratory physiotherapy planning and evaluation. In particular, LUS today represents an additional tool if performed by dedicated, long-trained physiotherapist. Its use, especially in intensive care unit [44], in addition to specific physiotherapeutic evaluation, should improve clinical management and patient care pathway and the development of personalized protocol. In fact, LUS provides information in the assessment of diaphragm kinetics and strength in patients with difficult weaning from mechanical ventilation. In particular, the use of ultrasound before and after pulmonary rehabilitation (PR) is an additional tool for the evaluation of diaphragm function in patients with Chronic Obstructive Pulmonary Disease (COPD) treated with PR. This provides a fast, reliable, non-invasive and relatively easy approach that allows repeated measurements and does not require patient effort or coordination, and should be used in combination with other examinations to evaluate PR results [45]. Some authors have focused on possible application of basic LUS by physiotherapists and how in clinical prac- tice [46]. LUS has better results than chest X-rays and clinical examination, so it should be considered as a useful tool in the physiotherapist's decision-making process [47].

Additionally, most recent Guidelines for the use of LUS by physiotherapists during the COVID-19 pandemic confirmed that physiotherapists could contribute to support medical team in the patient management of both acute and chronic illness and to guide their own physiotherapy interventions, too [48]. The absence of a specific physiotherapist-training program still remains an obstacle. On the other hand, a 2017 study has shown that is possible for physiotherapists without experience in LUS technique to acquire fundamental skills and become independent within 4-9 months [49].

\section{New protocol for cardiac rehabilitation patients}

The aim of this review is to suggest a standardize protocol for LUS in all the patients at the time of admission to Cardiac Rehabilitation Unit. Lung ultrasound should be performed in 6 scan each hemithorax (anterior, lateral and posterior scanning zones, divided by anterior and posterior axillary line, and upper and lower regions), covering 12 imagine regions, in all the patient in supine position, at rest. For the posterior scan, the patient can be seated or at 45 degrees, if there are no contraindications, allowing a better visualization of posterior recesses. (Figure 5).

For each scan, will be noted the specific physiological pattern or any pathological findings and written in the specific box (Figure 6):

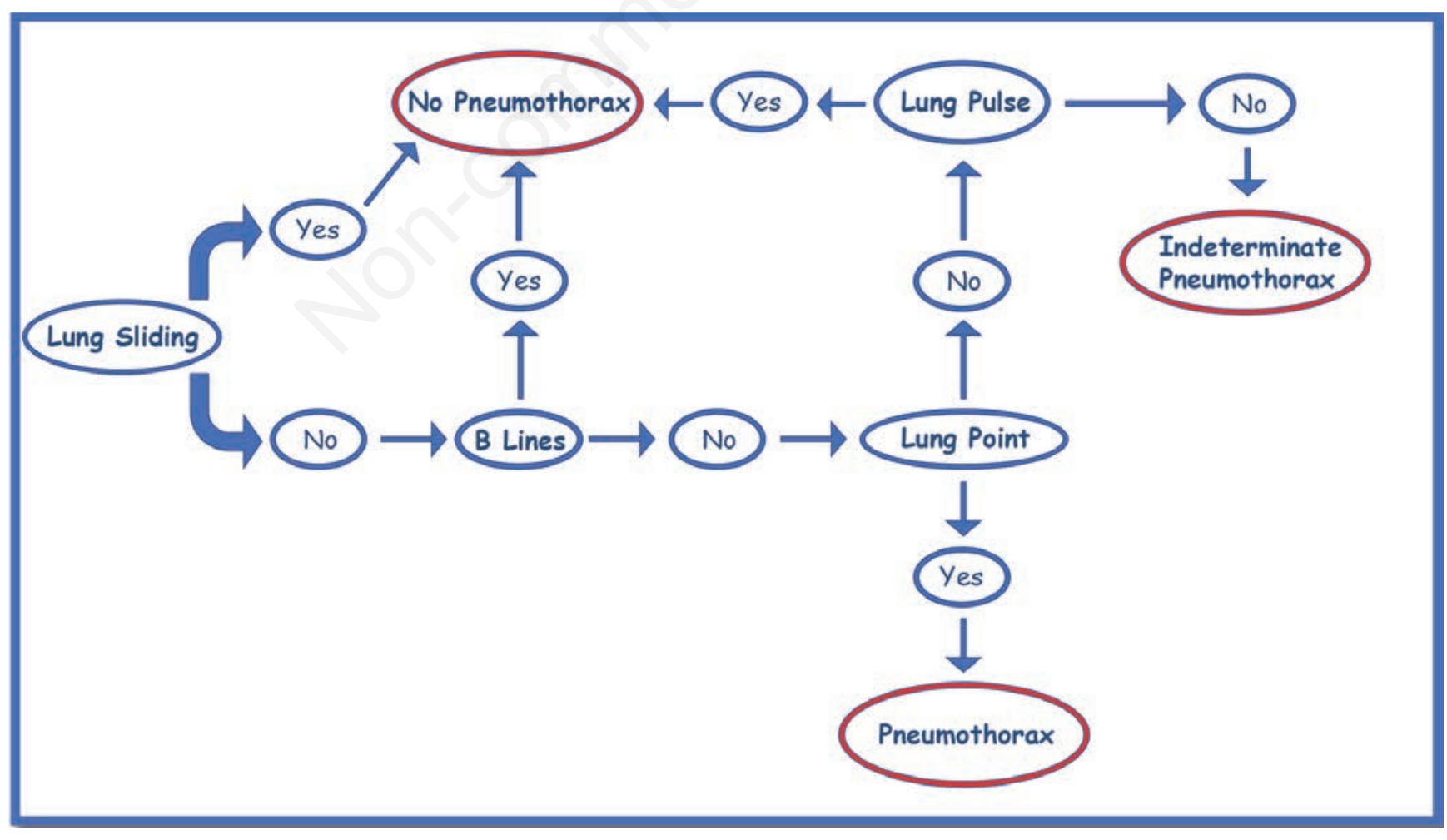

Figure 4. Flow chart on diagnosing pneumothorax. This flow chart suggests the correct sequence and combination of the four sonographic signs useful to rule out or rule in pneumothorax. 
- "A": A lines

- "B": B lines

- "AB": A + B lines

- "S+/S-": presence/absence of lung sliding

- "W": white lung

- "C": consolidation

- "Bs/Bd": static/dynamic air broncograms

- "Bf": fluid broncograms

- "P": lung pulse

- "Pt": lung point

- "PE": pleural effusion

Furthermore, we suggest the use of the lung ultrasound score (LUS Score) [50] for monitoring lung aeration. A LUS score of aeration can be calculated as follows: for each region of interest, points are allocated according to the worst ultrasound pattern observed:
- $0=($ pattern 0$)$ presence of A-lines beyond the pleural line: normal pulmonary aeration.

- $1=($ pattern 1) presence of multiple and well-separated vertical B-lines: moderate decrease in lung aeration resulting from interstitial syndrome.

- $2=($ pattern 2$)$ presence of coalescent B-lines: more severe decrease in lung aeration resulting from pulmonary edema or confluent bronchopneumonia

- $3=$ (pattern 3) presence of lung consolidation: complete loss of aeration with persisting aeration of distal bronchioles (dynamic bronchograms)

A LUS score ranging between 0 and 36 was calculated as the sum of each region. This score is a global view of lung aeration and can be regularly monitored. An increase in score indicates a more severe condition.

The final comment will be a comprehensive description of the pulmonary status.



Figure 5. Technique for LUS examination. Six regions of interest are examined on each side. A) Anterior (regions 1 and 2 ) and lateral lung regions (regions 3 and 4) delineated by the parasternal line (PSL), anterior axillary line (AAL) and posterior axillary line (PAL). B) Posterior lung regions (regions 5 and 6) delineated by the paravertebral line (PVL) and posterior axillary line. C) Posterior lung regions (regions 5 and 6 ) in patients who cannot be positioned in the seated position. To facilitate the ultrasound approach of posterior lung regions, patients should be positioned in the lateral decubitus position. 


\section{Azienda Ospedaliera Universitaria Federico II}

DAI DI EMERGENZE CARDIOVASCOLARI, MEDICINA CLINICA E DELL'INVECCHIAMENTO

U.O.C. CARDIOLOGIA RIABILITATIVA E CENTRO IPERTENSIONE

Responsabile: Prof. Nicola De Luca

\section{LUNG ULTRASOUND \\ Report Form}

Surname:

Name:

Room:

Bed:

Gender: $\square$ M $\square$ F Date of Birth: Storage Code:

Diagnosis:

Decubitus: $\square$ Supine $\square$ Lateral $\square$ Seated $/ \mathbf{4 5}^{\circ}$

BP: mmHg

HR:

$\mathrm{SpO}_{2:}$ $\%$

RR: min

\section{ABG}

pH: _ pCO $_{2}: \mathrm{HCO}^{3-}: \mathrm{PO}^{2}:$ - P/F:

$\mathrm{BE}:$ - $\mathrm{SpO}^{2}$ :

Hb:

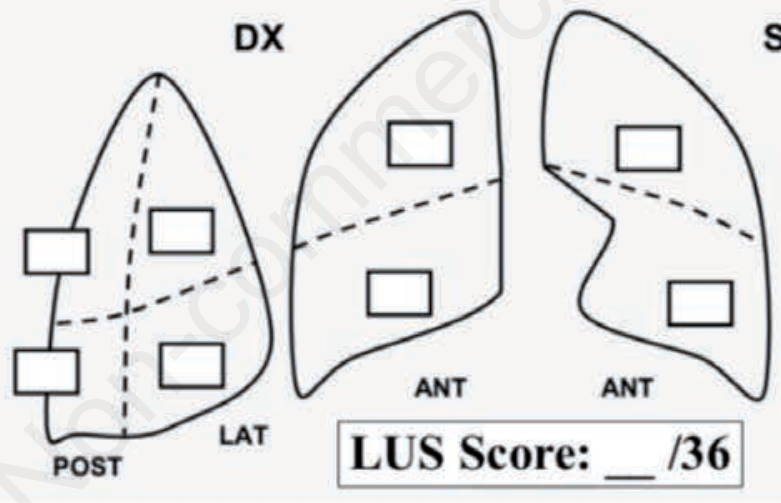

SN

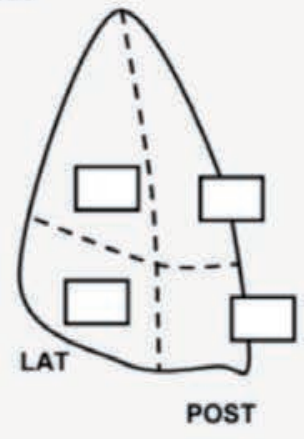

Legenda: A: A-lines; B: B-lines; AB: A+B lines; S+/S-: presence/absence of lung sliding; W: white lung; C: consolidation; Bs/Bd: static/dynamic air broncograms; P: lung pulse; Pt: lung point; PE: pleural effusion. LUS Score: $0=($ pattern 0$) ; 1=($ pattern 1$) ; 2=($ pattern 2$) ; 3=($ pattern 3$)$

\section{Description:}

\section{Diagnosis:}

Exam Date:

Operator:

Figure 6. Report form for lung ultrasound in Cardiac Rehabilitation Unit. 


\section{References}

1. Oks M, Cleven KL, Cardenas-Garcia J, et al. The effect of point-of-care ultrasonography on imaging studies in the medical ICU: a comparative study. Chest 2014;146:1574-7.

2. Mayo PH, Copetti R, Feller-Kopman D, et al. Thoracic ultrasonography: a narrative review. Intensive Care Med 2019; 45:1200-11.

3. Mancusi C, Carlino MV, Sforza A. Point-of-care ultrasound with pocket-size devices in emergency department. Echocardiography 2019;36:1755-64.

4. Sforza A, Mancusi C, Carlino MV, et al. Diagnostic performance of multi-organ ultrasound with pocket-sized device in the management of acute dyspnea. Cardiovasc Ultrasound 2017; $15: 16$.

5. Carlino MV, Paladino F, Sforza A, et al. Assessment of left atrial size in addition to focused cardiopulmonary ultrasound improves diagnostic accuracy of acute heart failure in the Emergency Department. Echocardiography 2018;35: 785-79.

6. Gargani L, Volpicelli G. How I do it: lung ultrasound. Cardiovasc Ultrasound 2014;12:25.

7. Sforza A, Carlino MV, Guarino M, et al. Anterior vs lateral symmetric interstitial syndrome in the diagnosis of acute heart failure. Int J Cardiol 2019; 280:130-2.

8. Sforza A, Carlino MV, Guarino M, et al. A case of pulmonary edema: The critical role of lung-heart integrated ultrasound examination. Monaldi Arch Chest Dis 2018;88:982.

9. Lichtenstein DA, Mezière GA. Relevance of lung ultrasound in the diagnosis of acute respiratory failure: the BLUE protocol. Chest 2008;134:117-25.

10. Lichtenstein DA. BLUE-protocol and FALLS-protocol: two applications of lung ultrasound in the critically ill. Chest 2015; 147:1659-70.

11. Lichtenstein D. Novel approaches to ultrasonography of the lung and pleural space: where are we now? Breathe Sheff Engl 2017;13:100-11.

12. Soldati G, Smargiassi A, Mariani AA, et al. Novel aspects in diagnostic approach to respiratory patients: is it the time for a new semiotics? Multidiscip Respir Med 2017;12:15.

13. Soldati G, Demi M, Smargiassi A, et al. The role of ultrasound lung artifacts in the diagnosis of respiratory diseases. Expert Rev Respir Med 2019;13:163-72.

14. Mohanty K, Blackwell J, Egan T, et al. Characterization of the lung parenchyma using ultrasound multiple scattering. Ultrasound Med Biol 2017; 43:993-1003.

15. Dietrich CF, Mathis G, Blaivas M, et al. Lung B-line artefacts and their use. J Thorac Dis 2016;8:1356-65.

16. Francisco MJ Neto, Rahal A Junior, Vieira FA, et al. Advances in lung ultrasound. Einstein (Sao Paulo) 2016;14:443-8.

17. Francis Chun Yue Lee. The curtain sign in lung ultrasound. J Med Ultrasound 2017;25:101-4.

18. Pedretti RFE, Fattirolli F, Griffo R, et al. Cardiac prevention and rehabilitation "3.0": from acute to chronic phase. Position paper of the Italian association for cardiovascular prevention and rehabilitation (GICR-IACPR). Monaldi Arch Chest Dis 2018;88:1004.

19. Prabhu NV, Maiya AG, Prabhu NS. Impact of cardiac rehabilitation on functional capacity and physical activity after coronary revascularization: a scientific review. Cardiol Res Pract 2020; 2020:1236968.

20. Brogi E, Bignami E, Sidoti A, et al. Could the use of bedside lung ultrasound reduce the number of chest x-rays in the intensive care unit? Cardiovasc Ultrasound 2017;15:23.

21. Platz E, Jhund PS, Girerd N, et al. Study group on acute heart failure of the acute cardiovascular care association and the heart failure association of the European society of cardiology. Expert consensus document: reporting checklist for quantification of pulmonary congestion by lung ultrasound in heart failure. Eur J Heart Fail.2019;21:844-85.

22. Martindale JL, Wakai A, Collins SP, et al. Diagnosing acute heart failure in the emergency department: a systematic review and meta-analysis. Acad Emerg Med 2016;23:223-42.

23. Soldati G, Demi M. The use of lung ultrasound images for the differential diagnosis of pulmonary and cardiac interstitial pathology. J Ultrasound 2017; 20:91-6.

24. Pivetta E, Goffi A, Nazerian P, et al. Study group on lung ultrasound from the Molinette and Careggi Hospitals. Lung ultrasound integrated with clinical assessment for the diagnosis of acute decompensated heart failure in the emergency department: a randomized controlled trial. Eur J Heart Fail 2019; 21:754-66.

25. Pivetta E, Goffi A, Lupia E, et al. SIMEU group for lung ultrasound in the emergency department in Piedmont. Lung ultrasound-implemented diagnosis of acute decompensated heart failure in the ED: a SIMEU multicenter study. Chest 2015;148:202-10

26. Ponikowski P, Voors AA, Anker SD, et al. ESC guidelines for the diagnosis and treatment of acute and chronic heart failure: the Task Force for the Diagnosis and Treatment of Acute and Chronic Heart Failure of the European Society of Cardiology. Eur J Heart Fail 2016;18:891-975.

27. Efremov SM, Kuzkov VV, Fot EV, et al. Lung ultrasonography and cardiac surgery: A narrative review. J Cardiothorac Vasc Anesth 2020; 34:3113-24.

28. Brogi E, Gargani L, Bignami E, et al. Thoracic ultrasound for pleural effusion in the intensive care unit: a narrative review from diagnosis to treatment. Crit Care 2017;21:325.

29. Balik M, Plasil P, Waldauf P, et al. Ultrasound estimation of volume of pleural fluid in mechanically ventilated patients. Intensive Care Med 2006;32:318.

30. Picano E, Scali MC, Ciampi Q, Lichtenstein D. Lung ultrasound for the cardiologist. JACC Cardiovasc Imaging 2018;11:1692-705.

31. Lazarevic A, Dobric M, Goronja B, et al. Lung ultrasoundguided therapeutic thoracentesis in refractory congestive heart failure. Acta Cardiol 2020;75:398-405.

32. Domokos D, SzaboA, Banhegyi G, et al. Needle aspiration for treating iatrogenic pneumothorax after cardiac electronic device implantation: a pilot study. J Interv Card Electrophysiol 2020;57:295-301.

33. Alrajab S, Youssef AM, Akkus NI, Caldito G. Pleural ultrasonography versus chest radiography for the diagnosis of pneumothorax: review of the literature and meta-analysis. Crit Care 2013;17:R208.

34. Volpicelli G. Sonographic diagnosis of pneumothorax. Intensive Care Med 2011;37:224-32.

35. Santos-Silva J, Lichtenstein D, Tuinman PR, Elbers PWG. The lung point, still a sign specific to pneumothorax. Intensive Care Med 2019;45:1327-8.

36. Lichtenstein DA, Menu Y. A bedside ultrasound sign ruling out pneumothorax in the critically ill: lung sliding. Chest 1995;108:1345-8.

37. Alzahrani SA, Al-Salamah MA, Al-Madani WH, et al. Systematic review and meta-analysis for the use of ultrasound 
versus radiology in diagnosing of pneumonia. Crit Ultrasound J 2017;9:6.

38. Reissig A, Copetti R, Mathis G et al. Lung ultrasound in the diagnosis and follow-up of community-acquired pneumonia: a prospective, multicenter, diagnostic accuracy study. Chest 2012;142:965-72.

39. Soldati G, Copetti R. Thoracic ultrasound. Turin, Edizioni medico-scientifiche; 2017.

40. Montero Baladía M, Arroyo Diez M, Badallo Areválo O. Basic sign in lung echography. Liquid and air bronchogram: differentiation. Med Intensiva 2018;42:19.

41. Asano M, Watanabe H, Sato K et al. Validity of ultrasound lung comets for assessment of the severity of interstitial pneumonia. J Ultrasound Med 2018; 37:1523-31.

42. Volpicelli G, Elbarbary M, Blaivas M, et al. International evidence-based recommendations for point-of-care lung ultrasound. Intensive Care Med 2012;38:577-91.

43. McKiernan S, Chiarelli P, Warren-Forward H. Diagnostic ultrasound use in physiotherapy, emergency medicine, and anaesthesiology. Radiography 2010;16:154-9.

44. Mojoli F, Bouhemad B, Mongodi S, Lichtenstein D. Lung ultrasound for critically ill patients. Am J Respir Crit Care Med 2019;199:701-14.

45. Crimi C, Heffler E, Augelletti T, et al. Utility of ultrasound assessment of diaphragmatic function before and after pulmonary rehabilitation in COPD patients. Int J Chron Obstruct Pulmon Dis 2018;13:3131-9.

46. Karthika M, Wong D, Nair SG, et al. Lung ultrasound: the emerging role of respiratory therapists. Respir Care 2019;64:217-29.

47. Le Neindre A, Mongodi S, Philippart F, Bouhemad B. Thoracic ultrasound: potential new tool for physiotherapists in respiratory management. A narrative review. J Crit Care 2016;31:101-9.

48. Hayward S, Duncan C. Physiotherapists use of lung ultrasound during the COVID-19 pandemic: a practical guideline on supporting acute hospital colleagues. Association of Chartered Physiotherapists in Respiratory Care; 2020.

49. Hayward S, Kelly D. Respiratory physiotherapy and lung ultrasound: a service evaluation of a training programme. J Assoc Chart Physiother Respir Care 2017; 50:51-58.

50. Soummer A, Perbet S, Brisson H, et al. Ultrasound assessment of lung aeration loss during a successful weaning trial predicts postextubation distress. Crit Care Med 2012;40:2064-72. 